\title{
Les coopératives agroalimentaires face aux enjeux de la mondialisation
}

\section{L'internationalisation des coopératives constitue-t-elle une solution? \\ Food-processing cooperatives faced with the challenges of globalization: Is internationalization a solution?}

\section{René Mauget}

Numéro 297, juillet 2005

XIX ${ }^{\mathrm{e}}$ Colloque de l'ADDES : Pour une économie conquérante et ouverte à ses partenaires

URI : https://id.erudit.org/iderudit/1021851ar

DOI : https://doi.org/10.7202/1021851ar

Aller au sommaire du numéro

\section{Éditeur(s)}

Institut de l'économie sociale (IES)

\section{ISSN}

1626-1682 (imprimé)

2261-2599 (numérique)

\section{Découvrir la revue}

Citer cet article

Mauget, R. (2005). Les coopératives agroalimentaires face aux enjeux de la mondialisation : l'internationalisation des coopératives constitue-t-elle une solution ? Revue internationale de l'économie sociale, (297), 42-59. https://doi.org/10.7202/1021851ar

\begin{abstract}
Résumé de l'article
Quelles stratégies les coopératives agroalimentaires adoptent-elles face à la mondialisation? Après une présentation du cadre conceptuel et du contexte de la mondialisation, l'auteur exploite d'une part des enquêtes réalisées depuis 1969 sur l'internationalisation des coopératives, d'autre part une banque de données sur les mouvements à l'international des entreprises agroalimentaires françaises de 1996 à 2003. Sur la dernière période, les coopératives sont moins présentes à l'international que les entreprises capitalistes - respectivement 20 $\%$ et $50 \%$ des opérations hors de France - et l'Europe concentre l'essentiel des mouvements. Datant de la fin des années 60 , ce développement à

l'international, qui privilégie la franchise et la filialisation, est principalement porté par onze groupes coopératifs. L'article propose une analyse qualitative de la démarche de ces groupes ainsi qu'une analyse par secteurs d'activité. Entre valeur pour l'usager et valeur pour l'actionnaire, les arbitrages sont souvent complexes. Les politiques publiques devront aussi considérer le fait que les coopératives jouent un rôle clé pour le maintien d'activités sur un territoire, comme en témoigne l'élargissement de leur périmètre en France.
\end{abstract}




\section{LES COOPÉRATIVES AGROALIMENTAIRES FACE AUX ENJEUX DE LA MONDIALISATION}

L'internationalisation des coopératives constitue-t-elle une solution?

(*) Professeur-chercheur, Ecole supérieure des sciences économiques et commerciales (Essec), Institut management international agroalimentaire (Imia). par René Mauget ${ }^{(*)}$

Quelles stratégies les coopératives agroalimentaires adoptent-elles face à la mondialisation? Après une présentation du cadre conceptuel et du contexte de la mondialisation, l'auteur exploite d'une part des enquêtes réalisées depuis 1969 sur l'internationalisation des coopératives, d'autre part une banque de données sur les mouvements à l'international des entreprises agroalimentaires françaises de 1996 à 2003. Sur la dernière période, les coopératives sont moins présentes à l'international que les entreprises capitalistes - respectivement $20 \%$ et $50 \%$ des opérations hors de France - et l'Europe concentre l'essentiel des mouvements. Datant de la fin des années 60, ce développement à l'international, qui privilégie la franchise et la filialisation, est principalement porté par onze groupes coopératifs. L'article propose une analyse qualitative de la démarche de ces groupes ainsi qu'une analyse par secteurs d'activité. Entre valeur pour l'usager et valeur pour l'actionnaire, les arbitrages sont souvent complexes. Les politiques publiques devront aussi considérer le fait que les coopératives jouent un rôle clé pour le maintien d'activités sur un territoire, comme en témoigne l'élargissement de leur périmètre en France.
(1) OMC: Organisation mondiale du commerce. Pac: politique agricole commune. ace à la mondialisation des marchés et à l'internationalisation des entreprises capitalistes, les groupes coopératifs s'interrogent sur les stratégies à conduire pour pérenniser leurs activités et valoriser au mieux leurs productions sur leur territoire. Doivent-elles par mimétisme ou par intérêt suivre le même chemin que les entreprises multinationales capitalistes ou doivent-elles rester sur leur bassin de production?

A ce jour, nous avons répertorié dans nos enquêtes près de 450 mouvements ou opérations d'internationalisation depuis trente-cinq ans (1969-2004). Sur environ 3500 coopératives aujourd'hui (hors Cuma), une cinquantaine seulement ont effectué des manœuvres à l'international (hors exportation).

\section{Le contexte}

La globalisation des marchés avec la réduction des protections est engagée et va sans doute s'accélérer à la suite des prochains accords OMC-Pac ${ }^{(1)}$. Conjointement, nous assistons à une internationalisation croissante des entreprises capitalistes agroalimentaires (investissements directs à l'étranger). 
(2) Etude Essec-Imia, 1996-2003.

Ces dernières années, plus de $50 \%$ des opérations d'alliance ou de fusionacquisition partant des entreprises françaises capitalistes ont été effectuées avec l'étranger. Pour les coopératives, cette stratégie est nettement moins importante $(20 \%)^{(2)}$.

Le comportement des coopératives est-il différent de celui des entreprises capitalistes sur ce point?

Dans ce nouveau contexte encore mal régulé, quelles places, quelles stratégies et quels résultats pour les coopératives agricoles et agroalimentaires face à ces enjeux?

On aurait pu craindre depuis quelques années une réduction du périmètre des coopératives face à cette globalisation des marchés et à l'internationalisation des concurrents capitalistes, or depuis sept ans (1997-2003), le périmètre des groupes coopératifs agroalimentaires a augmenté de quelques points (2 à $3 \%)$ en France.

Ce phénomène s'explique notamment par un désengagement du capital agroalimentaire et surtout des fonds de pension, capital repris par des coopératives.

Cette stratégie des groupes coopératifs s'inscrit dans la logique du développement durable dans les territoires (revenu et emploi).

Cependant, si la mondialisation libérale s'accentue, la capacité de compensation des coopératives sera insuffisante dans de nombreux secteurs (lait, volailles, légumes, conserves, etc.) pour faire face à ce phénomène. Une régulation européenne est donc indispensable si l'on veut éviter des faillites qui auront des conséquences néfastes sur les territoires.

\section{Les hypothèses de recherche}

- Les coopératives bénéficieront moins des avantages de l'internationalisation ou de la délocalisation (finalité orientée sur la valorisation du produit) que les entreprises capitalistes (finalité orientée sur la rentabilité des capitaux).

- L'accroissement de valeur pour l'actionnaire ne compense souvent pas la perte de valeur apparente pour l'usager dans l'internationalisation des coopératives.

- Les coopératives s'impliqueront plus que les entreprises capitalistes dans la pérennité des bassins de production.

\section{Les concepts utilisés pour l'analyse}

Nous introduirons d'abord les différents concepts nécessaires à l'analyse:

- concepts pour définir la différence entre l'entreprise coopérative et l'entreprise capitaliste;

- concepts de valeurs pour l'usager et de valeurs pour l'actionnaire dans l'entreprise coopérative.

\section{Théorie de l'agence revisitée et différenciation des entreprises} (G. Deshayes, 1988)

Dans toutes les entreprises il existe des facteurs risqués et des facteurs contractuels. Donc, les organisations économiques passent deux types de contrats: 
(3) Q: quantité. PP: prix des produits. Qch: quantité des charges (engrais, phyto, services).
- à prix non fixe (risqué) avec les ayants droit;

- à prix fixe avec les autres agents économiques.

Les ayants droit assument le risque de l'activité économique de l'organisation en contractant avec l'agence à prix incertain.

Ainsi, au regard de cette approche, on arrive à deux conclusions:

- Les coopératives sont comparables dans leur construction aux entreprises capitalistes (le facteur risqué donne le pouvoir sur le surplus).

- Les coopératives agricoles sont profondément différentes. Le facteur risqué n'est pas le même, ce qui engendre des différences sur les critères d'efficacité et sur la pertinence de telles ou telles manœuvres stratégiques: - dans la coopérative, le facteur risqué égale le produit;

- dans la firme capitaliste, le facteur risqué égale le capital.

Les implications stratégiques et financières de ces conclusions sont déterminantes, notamment dans les stratégies d'internationalisation et plus encore de délocalisation des productions agricoles et industrielles.

\section{Apport de valeur par la coopérative pour l'usager}

Nous prendrons l'exemple d'une coopérative d'écoulement (collecte et transformation).

\section{Avantage court terme sur le revenu}

La coopérative peut intervenir sur le revenu de trois manières:

- avantages prix (prix coopératif-prix de marché);

- avantages quantité (droit à produire, effet de productivité sur l'exploitation agricole);

- avantages par la maîtrise des charges sur les exploitations agricoles.

Revenu $=(\mathrm{Q} \times \mathrm{PP}-\mathrm{Q} \text { ch } \times \mathrm{Pch})^{(3)}$

\section{Sécurité paiement}

Il est en effet très rare qu'en cas de faillite ou de difficultés l'engagement de paiement des produits par la coopérative ne soit pas respecté. La coopérative est le plus souvent reprise par une autre coopérative qui respecte les engagements de paiement. Il n'en est pas toujours de même dans le cas d'une faillite d'entreprise non coopérative.

\section{L'avantage coopératif à long terme}

L'avantage coopératif se manifeste à long terme par un moindre risque de délocalisation de la production, donc du revenu de l'agriculteur. En effet, les critères de décision sont différents de l'entreprise non coopérative (ENC). Pour une ENC multirégionale ou multinationale, le raisonnement rationnel est le suivant: peut-on trouver une meilleure valorisation de capitaux sur le long terme en délocalisant l'activité? Voir aussi la notion de risque. Dans le cas de l'entreprise coopérative, la délocalisation, peu fréquente, ne s'effectue qu’après avoir épuisé les solutions économiques dans la région d'origine (l'avantage pour l'usager en termes de revenu l'emporte sur la notion de rentabilité des capitaux découlant d'une éventuelle délocalisation). 
(4) VC: valeur créée (economic value added, en anglais). $\mathrm{RCI}$ : rentabilité des capitaux investis en pourcentage (résultat opérationnel moins l'impôt). CE: coût des capitaux engagés en pourcentage. $\mathrm{Cl}$ : capitaux investis, soit valeurs immobilisées nettes plus BFR.
Néanmoins, dans le cadre de raisonnements complexes, le groupe coopératif peut être amené à effectuer son expansion en dehors de son territoire, mais ce n'est que rarement une décision de premier choix.

\section{Apport de valeur par le groupe coopératif pour l'actionnaire}

Nous définirons d'abord ce concept dans le cadre de l'entreprise capitaliste, puis nous verrons si la même approche peut être retenue dans les groupes coopératifs complexes.

La valeur créée par une entreprise capitaliste correspond à la différence entre la rentabilité des capitaux investis et le coût des capitaux engagés. $\mathrm{VC}=(\% \mathrm{RCI}-\% \mathrm{CCE}) \times \mathrm{CI}^{(4)}$

La façon d'appréhender ce concept dans un groupe coopératif est plus complexe.

\section{Cas d'une filiale qui transforme des produits ne provenant pas des associés (filiale délocalisée, par exemple)}

Dans ce cas de figure, le raisonnement que nous avons évoqué ci-dessus peut être pertinent à condition que cet investissement n'ait pas d'effet négatif sur la valeur, dans le groupe coopératif en France, pour l'usager.

\section{Cas d'une filiale qui transforme les produits des sociétaires en France}

Dans ce cas, il convient d'étudier la façon dont a été déterminé le prix de marché pour le produit de base. Si celui-ci a été convenablement évalué par rapport à l'environnement, le raisonnement précédent peut encore être pertinent.

\section{Valeur pour la coopérative ou valeur pour l'adhérent?}

Sur le long terme, l'adhérent demande la pérennité et l'augmentation de ses "droits à produire " et de son revenu.

Sur le court terme, l'agriculteur dans la coopérative classique était intéressé par un sur-prix ou un sur-revenu en provenance de sa coopérative par rapport à l'agriculteur qui travaillerait avec une entreprise non coopérative (dans la réalité, ce n'est pas toujours le cas). Donc, l'organisation en groupe complexe filialisé doit permettre de verser à l'associé coopérateur un montant au moins équivalent au montant quil percevrait dans la coopérative classique. Cependant, celui-ci sera versé sous une forme différente, à savoir : prix de marché (PM) plus dividendes avec avoir fiscal (DAF) versés au prorata du capital détenu par le sociétaire dans sa coopérative (ceci est possible depuis la loi du 3 janvier 1991 en France). Dans la « moulinette » coopérative, on peut cependant faire un mixage des modes de rémunération de l'agriculteur usager et actionnaire.

Ainsi, la valeur du groupe coopératif pour le " coopérateur actionnaire " à court terme se limite au pourcentage de dividendes versés par rapport au capital détenu par lui, ce montant s'ajoutant au prix qu'il aura perçu antérieurement. 
(5) Possibilité cependant de revaloriser le capital social (en France) en fonction du barème des rentes viagères (loi de 1972).
(6) Voir Recma, n 278-279, " De la coopérative au groupe coopératif agroalimentaire ", de R. Mauget et M. Forestier, qui traite ce sujet.
A cela peut s'ajouter la rémunération du capital social du sociétaire qui, nous l'avons dit, pourrait se situer au niveau des obligations sans risque comme dans la coopérative classique, pour que cette variable soit neutralisée dans la comparaison.

Nous rappelons que l'associé coopérateur n'a pas, en théorie, de droits sur les réserves ${ }^{(5)}$.

L'agriculteur peut cependant avoir accès à des plus-values si ce dernier investit directement dans la filiale délocalisée.

Il en résulte que l'analyse de l'avantage coopératif pour l'usager et (ou) pour l'actionnaire dans un groupe coopératif est très complexe.

C'est dans cette complexité que se situe le choix d'internationalisation, et l'optimisation de cette stratégie sera différente selon que l'on regarde les valeurs créées au niveau du groupe coopératif ou au niveau des adhérents.

\section{L'internationalisation ou la délocalisation des groupes coopératifs}

Est-ce pertinent?

Dans la pratique, on pourrait concevoir une stratégie de délocalisation ou d'expansion hors région (c'est déjà une réalité dans certaines productions) qui permettrait de bénéficier de faibles coûts d'intrants et de salaires pour augmenter les résultats, lesquels seraient ensuite distribués aux adhérents du groupe coopératif, au prorata du capital social. La valeur pour l'actionnaire prendrait ainsi une place plus importante, mais celle-ci serait à comparer à une éventuelle diminution des « droits à produire " pour les adhérents dans leur région ou pays d'origine, donc une perte pour l'usager.

Dans le cadre de la globalisation des marchés et de l'internationalisation des concurrents, cette réflexion devient pertinente pour l'agriculteur " actionnaire " et usager. Faut-il s'implanter en Chine pour produire du malt? Faut-il aller faire du foie gras en Bulgarie, pour le réimporter en UE? Faut-il aller produire du poulet au Brésil pour les marchés du Moyen-Orient ou d'ailleurs? Etc. Cela est possible, mais est-ce toujours pertinent par rapport aux finalités?

La réflexion par rapport aux principes coopératifs serait également pertinente, mais nous ne traiterons pas cet aspect dans cette contribution ${ }^{(6)}$.

\section{La mondialisation des échanges et l'internationalisation des entreprises s'accélèrent}

Nous distinguerons trois logiques d'internationalisation des activités des entreprises:

- l'exportation de produits;

- l'internationalisation par implantation industrielle et commerciale pour servir le marché local;

- la délocalisation industrielle pour réexpédier la production en France ou en Europe.

De même, trois critères de mondialisation des marchés et des entreprises peuvent être analysés. 


\section{Evolution des exportations mondiales}

En moyenne, depuis vingt-cinq ans, la croissance des exportations mondiales est deux fois plus importante que la croissance du PIB mondial. Nous notons une accélération de $12 \%$ au cours de l'année 2000 et une chute en 2001, mais en 2003 la croissance est repartie.

Sur une longue période, on observe également le déclin des pays de l'OCDE au profit de l'Asie (hors Japon).

En matière d'évolution des exportations de produits agroalimentaires, la France a connu de fortes progressions de ses exportations de 1975 à 1997. Depuis quelques années, les exportations ont stagné et le solde de la balance commerciale est en baisse après avoir connu un pic en 1997.

Dans ce contexte, les coopératives agricoles sont et vont être affectées directement, car l'exportation constituait pour elles des débouchés sur des produits basiques, lesquels seront les plus touchés (vins, poulets exports, beurre et poudre de lait, etc.).

Par ailleurs, nous verrons que les stratégies de délocalisation ne sont pas souvent en cohérence avec les finalités de la coopérative.

Les investissements directs à l'étranger (IDE)

Au niveau mondial (tous secteurs)

Année

1995

2000

2001

2003

Milliards de dollars

300

1300

760

Ces derniers sont également en augmentation parfois forte (2000). Ce deuxième critère est intéressant pour quantifier les efforts d'internationalisation et de délocalisation des entreprises. La Chine, ces dernières années, arrive en numéro un comme récepteur d'IDE.

Nous verrons dans un prochain chapitre que le secteur agroalimentaire suit largement cette dynamique, mais que les coopératives sont peu présentes en matière d'IDE.

\section{La population adhérente à l'OMC et les soutiens aux agricultures}

Ce critère est également important, car l'adhésion implique une baisse des protections (droits de douane, prélèvements, restitutions, etc.) et favorise donc la croissance des échanges et une relative spécialisation des pays. L'entrée de la Chine ces dernières années, puis demain celle de la Russie constituent des leviers d'accélération de cette mondialisation.

Dans ce concert de globalisation des marchés et d'internationalisation des concurrents, nous verrons que les coopératives constituent un facteur de stabilisation des bassins de production, mais que la capacité de résistance face aux pays émergents sera limitée si tous les droits de douane, 
prélèvements et autres aides directes venaient à disparaitre (voir tableau 1). Les soutiens prennent des formes diverses:

- barrières à l'entrée (prélèvements);

- aides à l'exportation (restitutions);

- sur-paiement des consommations en raison des quotas (lait, sucre);

- aide à l'hectare ou aux produits; etc.

Demain les restitutions vont disparaitre sur pression de l'OMC, mais le maintien, dans certains secteurs, des prélèvements ou des aides directes (à l'hectare ou aux produits) reste indispensable pour éviter une délocalisation à grande échelle des productions de masse. Le modèle néo-zélandais est certes un exemple intéressant de disparition des aides depuis 1984, mais les fondamentaux de cette économie sont différents de ceux de l'UE. Le modèle brésilien, aujourd'hui grand concurrent de l'Europe (sucre, volailles, porcs...), est encore plus redoutable pour les dix prochaines années.

Dans ce contexte, il n'y a cependant pas que des risques, les pays émergents, qui représentent plus de $50 \%$ de la population mondiale, pouvant être source de débouchés pour certains produits agroalimentaires.

\section{Tableau 1}

\section{Soutiens en 2000}

Estimation du soutien aux producteurs agricoles par pays en pourcentage de la valeur des recettes agricoles brutes

Année 2000

\begin{tabular}{|c|c|}
\hline Union européenne. & 34 \\
\hline République tchèque & .17 \\
\hline Hongrie.. & 22 \\
\hline Pologne... & 15 \\
\hline Slovaquie. & 25 \\
\hline Islande . & 64 \\
\hline Norvège. & 68 \\
\hline Suisse .... & .72 \\
\hline Turquie. & 21 \\
\hline Canada & 19 \\
\hline Etats-Unis & .22 \\
\hline Mexique. & .24 \\
\hline Japon & 60 \\
\hline Australie. & .5 \\
\hline Nouvelle-Zélande. & \\
\hline OCDE & 32 \\
\hline
\end{tabular}

Source: Déméter, 2004, éd. Armand Colin. 


\section{Les stratégies des coopératives françaises à l'international}

(7) Par manœuvres, nous entendons toutes opérations à l'international hors exportation directe, mais en intégrant les filiales commerciales.
(8) Cette banque de données ne prend en compte que les groupes agroalimentaires. Elle exclut donc l'agrofourniture et les semenciers.
Nous allons analyser la place et le comportement des groupes coopératifs français dans cet univers au regard des finalités de ces groupes.

Ce chapitre repose sur plusieurs informations:

- Une banque de données sur les manœuvres stratégiques ${ }^{(7)}$ des entreprises agroalimentaires françaises dans leurs relations avec les entreprises des autres pays de 1996 à 2003 (coopératives et entreprises capitalistes) nous permettra d'effectuer des comparaisons entre les deux types d'entreprises. Elle comporte plus d'un millier d'opérations dont $50 \%$ à l'international pour les entreprises capitalistes et $20 \%$ seulement pour les entreprises coopératives. - En plus de cette banque de données, nous avons effectué des enquêtes plus exhaustives de 1969 à 2004 sur l'internationalisation des coopératives, en intégrant, en plus des entreprises agroalimentaires, les coopératives de collecte de céréales, d'agrofourniture et de semences. Cette deuxième base de données intègre près de 450 opérations sur trente-cinq ans. Avant 1970, il n'y avait pratiquement pas d'opérations de coopératives à l'international (hors export).

\section{Les coopératives et la mondialisation (base IAA 1996-2004)}

On notera principalement six modalités de participation au concert de la mondialisation pour les coopératives:

- l'exportation (cet aspect sera évoqué, mais non analysé);

- les filiales ou participations commerciales à l'étranger;

- les filiales ou participations industrielles et commerciales à l'étranger;

- le transfert de technologie et la recherche;

- la franchise;

- autres, divers.

Les firmes capitalistes sont plus internationalisées que les coopératives et avec des formes différentes. Pour effectuer cette comparaison, nous utiliserons la banque de données Essec-Imia sur la période 1996-2003 ${ }^{(8)}$ (43 opérations d'entreprises coopératives, 400 opérations d'entreprises capitalistes).

Part des opérations stratégiques à l'internationalisation

\begin{tabular}{|lcccccccc|}
\hline En \% & 1996 & 1997 & 1998 & 1999 & 2000 & 2001 & 2002 & 2003 \\
\hline Groupes coopératifs français & 20 & 23 & 17 & 40 & 18 & 32 & 6 & 8 \\
Entreprises capitalistes françaises & 44 & 36 & 39 & 52 & 66 & 62 & 55 & 35 \\
\hline
\end{tabular}

On observe sur cette période que les coopératives ont effectué environ $20 \%$ de leurs mouvements stratégiques à l'international, alors que les entreprises capitalistes ont réalisé plus de 50 \% de leurs manœuvres hors de France sur cette période. 
Cette différence importante prend sa source dans les finalités premières. Pour la coopérative, il s'agit de valoriser les produits des sociétaires. Pour l'entreprise capitaliste, le but est de valoriser des capitaux et, dans le contexte actuel, l'internationalisation est souvent une variable clef de la croissance et de la rentabilité à moyen terme et parfois à court terme.

La difficulté des coopératives pour mobiliser des capitaux constitue également une seconde raison.

Localisation des opérations internationales

\begin{tabular}{|lccccccc|}
\hline$\%$ & $\begin{array}{c}\text { Europe } \\
\text { occidentale }\end{array}$ & $\begin{array}{c}\text { PECO, } \\
\text { Russie }\end{array}$ & $\begin{array}{c}\text { Asie, } \\
\text { Pacifique }\end{array}$ & $\begin{array}{c}\text { Amérique } \\
\text { du Sud }\end{array}$ & $\begin{array}{c}\text { Maghreb, } \\
\text { pays arabes }\end{array}$ & $\begin{array}{c}\text { Afrique } \\
\text { Amérique } \\
\text { du Nord }\end{array}$ \\
\hline Coopératives & 63 & 16 & 7 & 5 & 5 & 5 & 0 \\
Capitalistes & 39 & 15 & 13 & 11 & 5 & 5 & 12 \\
\hline
\end{tabular}

L'essentiel des opérations des entreprises coopératives se situe en Europe : 80 \% (y compris PECO). L'Amérique du Nord est absente des opérations coopératives durant cette période. Il existe cependant des opérations de franchise menées par Yoplait, mais elles sont plus anciennes, avec General Mills aux Etats-Unis et Agropur au Canada.

L'internationalisation des groupes capitalistes est par contre plus large, puisque l'Europe, y compris les PECO et la Russie, ne représente que $54 \%$ des opérations. L'Asie est beaucoup plus présente et l'Amérique du Nord également.

La nature des opérations est également différente

\begin{tabular}{|c|c|c|c|}
\hline$\%$ & $\begin{array}{l}\text { Rachat } \\
\text { et PPM* }\end{array}$ & $\mathbf{J V}^{* *}$ & $\begin{array}{l}\text { Autres formes + accord commerciaux } \\
+ \text { transfert techno.-franchise }\end{array}$ \\
\hline Coopératives & 40 & 23 & 37 \\
\hline Capitalistes & 56 & 12 & 32 \\
\hline
\end{tabular}

Les coopératives font moins de rachats et de prises de participation majoritaires compte tenu d'une plus grande difficulté à mobiliser des capitaux et des finalités de la coopérative. Les accords de franchise et les filiales commerciales sont par contre plus développés dans l'échantillon des coopératives. Cette stratégie est cohérente pour les coopératives. En effet, les filiales commerciales sont en phase avec les finalités de la coopérative, puisqu'elles permettent d'exporter des produits des agriculteurs français, notamment. La franchise, quant à elle, ne demande pas d'investissements élevés et est donc 
compatible avec la difficulté à mobiliser des capitaux; le développement de la Sodiaal (Yoplait) à l'international a été effectué essentiellement par cette stratégie.

Nous verrons cependant que les coopératives ont effectué des opérations à plus fort coefficient de capital, notamment dans le sucre et le malt.

\section{Conclusion sur cette comparaison}

L'intensité, les modalités, les motivations de l'internationalisation ne sont pas de même nature et de même degré pour les groupes coopératifs et pour les groupes capitalistes. L'essentiel de l'explication réside dans la différence de facteur risqué de la société mère, pour l'une le produit, pour l'autre le capital.

\section{Analyse élargie (base 1969 à 2004; voir tableau 2)}

Dans cette seconde partie de l'analyse, nous utiliserons nos enquêtes sur trente-cinq ans, enquêtes élargies aux secteurs de l'agrofourniture et surtout aux coopératives semencières qui ont été actives dans l'internationalisation. Au total, 444 opérations ont été enregistrées depuis 1969 jusqu’à 2004. Sur cette même période, nous avons enregistré 64 opérations de cessions ou d'arrêt d'activité, soit $15 \%$, parfois suite à des faillites; par exemple, ULN, reprise par le groupe capitaliste Bongrain au début des années 90, ou France Champignon, repris par une société d'investissement en 2004. Linternationalisation des groupes coopératifs français est limitée et récente (fin des années 60). Il s'agit essentiellement de la Sodiaal, alors appelé Sodima, avec la franchise de la marque Yoplait (première franchise en Suisse en 1969).

\section{Tableau 2}

Part de chacun des secteurs dans le total des opérations internationales (444 opérations, 35 groupes coopératifs impliqués)

$\% \quad$ Nombre de coopératives

$\begin{array}{lrr}\text { Semenciers } & 41,2 & 4 \\ \text { Lait } & 30,4 & 7 \\ \text { Industrie du grain } & 9,2 & 3 \\ \text { Polyvalent } & 5,6 & 4 \\ \text { Fruits et légumes } & 5,2 & 6 \\ \text { Vin } & 3,8 & 7 \\ \text { Sucre } & 3,2 & 2 \\ \text { Viande } & 1,4 & 2 \\ \text { Total } & \mathbf{4 4 4} & \mathbf{3 5}\end{array}$


Ensuite, le développement à l'international a été effectué par les semenciers, notamment Limagrain, puis dans les années 90 par les sucriers, à l'exemple de SDA, aujourd'hui Tereos, puis par les malteurs essentiellement (Malteurop, filiale de coopératives céréalières autour de Champagne Céréales).

En 2004, plus de $80 \%$ des opérations à l'international sont effectuées par 11 groupes coopératifs sur les 3500 coopératives dénombrées en France. Deux groupes, Sodiaal et Limagrain, représentent le tiers des mouvements à l'étranger.

Il convient de préciser que les groupes coopératifs internationalisés sont souvent constitués autour d'une union de coopératives, par exemple In Vivo (plusieurs centaines de coopératives adhérentes), Sodiaal (plusieurs dizaines), ULN (aujourd'hui disparue). Champagne Céréales, actionnaire de référence, est associé à quelques dizaines de coopératives dans Malteurop et Nutrixo.

\section{Analyse par secteur d'activité (tableau 2)}

Deux secteurs dominent.

- Le secteur semences vient en première position, avec plus de $41 \%$ des opérations et quatre groupes dans le top 11 ci-après (tableau 3).

- Le secteur laitier représente plus de $30 \%$ des opérations avec sept entreprises; parmi celles-ci, deux groupes ont été repris par le groupe Bongrain (ULN et Bressor) et 3A vient de céder en 2004 à Lactalis trois filiales espagnoles.

Les autres secteurs sont plus récents dans leur internationalisation.

- Le vin : sept groupes coopératifs dont Val d'Orbieu.

- L'industrie des grains, essentiellement à partir des filiales Malteurop et Nutrixo.

- Les fruits et légumes, où six coopératives sont impliquées, dont le groupe Avril, qui a été repris dans les années 90. Les groupes Cecab et Agrial, avec respectivement les marques D'Aucy et Florette, souhaitent développer leur implantation européenne, jusqu'en Russie pour la Cecab. La coop de Cerno, quant à elle, petite coopérative du Périgord, a effectué une délocalisation partielle en Pologne.

- Les coopératives polyvalentes présentes essentiellement dans l'ouest de la France n'ont pas eu une activité internationale débordante; elles sont plus orientées sur le développement dans leur territoire (quatre groupes impliqués, essentiellement des filiales commerciales).

- Les coopératives sucrières (Tereos et Cristal Union, qui ont repris l'essentiel de Béghin-Say) sont plus actives à l'international, notamment vers les PECO et, pour Tereos, vers le Brésil, afin de récupérer les marchés mondiaux qui seront perdus après la réforme de la Pac dans les prochaines années.

- Enfin, dans le secteur viande, les entreprises Socopa et Cooperl n'ont pas eu une stratégie très offensive à l'international jusqu'à ce jour.

Pour résumer, on peut noter que quelques entreprises dans chaque secteur ont développé une stratégie délibérée d'internationalisation, 
notamment dans les activités suivantes: lait; semences; sucre; grains transformés (malt, farine); et, plus récemment, dans les légumes.

Aujourd'hui, face à la crise viticole, quelques entreprises recherchent des solutions dans des accords internationaux, à l'exemple du groupe Val d'Orbieu avec Caviro, en Italie, ou de la coopérative de Limoux avec l'américain Gallo en 2004.

Les filiales commerciales et la franchise représentent une part importante des opérations avec l'étranger.

\section{Analyse qualitative à partir du top 11 (tableau 3)}

Nous rappelons que le top 11 intègre plus de $80 \%$ des opérations d'internationalisation des entreprises coopératives.

Les onze groupes coopératifs les plus actifs en nombre d'opérations à l'international se situent dans les vingt premiers groupes coopératifs français.

\section{La Sodiaal (Yoplait-Candia), 2,474 milliards d'euros (2002)}

Le groupe a engagé son internationalisation dès la fin des années 60 avec la marque Yoplait.

Aujourd'hui, le groupe est présent dans une quarantaine de pays et serait en position de numéro deux mondial sur les produits frais derrière Danone, avec une stratégie essentiellement orientée vers la franchise (plus des deux tiers des opérations).

Le groupe a également tenté la stratégie d'implantation industrielle, mais cette stratégie a connu quelques revers, avec des fermetures d'usines ou des reventes, notamment en Espagne et en Pologne (aujourd'hui, le groupe possède quelques sites industriels en Europe).

Au début des années 2000, les résultats bénéficiaires sur les activités franchisées étaient à peu près équivalents aux pertes enregistrées sur les implantations industrielles.

Avec la marque Candia sur le lait, le groupe a également effectué des opérations internationales par franchise et par implantation industrielle, mais de façon plus réduite.

Dans l'activité "fromage », le groupe possède aussi quelques filiales en Europe.

\section{ULN (Union laitière normande)}

A la fin des années 70, ce groupe était au premier rang des coopératives françaises.

Dans les années 80, il s'est lancé dans une stratégie d'implantation industrielle et commerciale par rachat aux Etats-unis, en Espagne, en Russie, en Belgique..., à une période où les taux d'intérêts réels étaient élevés.

L'internationalisation n'est sans doute pas la seule cause de la chute du groupe repris par Bongrain, mais cette stratégie, beaucoup plus risquée que celle de Sodiaal par la franchise, a contribué incontestablement à la chute du groupe. Aujourd'hui, les anciennes coopératives adhérentes de l'ULN possèdent une participation minoritaire dans la filiale (CLE) du groupe Bongrain. 


\section{Eurial et $3 \mathrm{~A}$}

Eurial et $3 \mathrm{~A}$ sont des groupes laitiers, anciens adhérents du groupe Sodiaal, qui ont préféré prendre leur autonomie lorsque la Sodiaal a restructuré le groupe avec filialisation par métiers. Ces deux groupes possèdent une activité internationale.

- 3A: 1,027 milliard d'euros (2002); basé à Toulouse, a effectué son internationalisation essentiellement sur l'Espagne, stratégie industrielle et commerciale. En 2004, le groupe, suite à des difficultés financières, a revendu l'essentiel de ses filiales industrielles à Lactalis, numéro un laitier français. - Eurial-Poitouraine: 0,484 milliard d'euros (2002). Ce groupe a quelques implantations industrielles en Pologne et en Espagne et des partenariats commerciaux.

\section{Coopagri Bretagne}

Groupe polyvalent de Bretagne, ce groupe apparaît à ce rang grâce à la filiale Laïta, notamment, commune avec Even et Terrena, filiale de commercialisation de produits laitiers.

\section{Groupe Champagne Céréales et coopératives associées} (0,989 milliard d'euros pour Champagne Céréales 2002-2003)

Ce groupe est actionnaire principal de deux filiales internationalisées:

- Malteurop (malt);

- Nutrixo (farine).

La logique d'internationalisation a été pour Malteurop de suivre les brasseurs pour ne pas être marginalisé.

\section{Tableau 3}

\section{Le top 11 à l'international}

\begin{tabular}{|c|c|c|c|}
\hline Sodiaal &. .78 & $21,3 \%$ & $17,6 \%$ \\
\hline Limagrain & 64 & $17,4 \%$ & $14,4 \%$ \\
\hline In Vivo & 61 & $16,6 \%$ & $13,7 \%$ \\
\hline Maïsadour & 42 & $11,4 \%$ & $9,5 \%$ \\
\hline Champagne Céréales* & 32 & $8,7 \%$ & $7,2 \%$ \\
\hline ULN. & .23 & $6,3 \%$ & $5,2 \%$ \\
\hline Coopagri-Bretagne ${ }^{* *}$ & .17 & $4,6 \%$ & $3,8 \%$ \\
\hline Euralis & .16 & $4,4 \%$ & $3,6 \%$ \\
\hline Tereos & .12 & $3,3 \%$ & $2,7 \%$ \\
\hline Eurial . &. .11 & $3,0 \%$ & $2,5 \%$ \\
\hline Cecab. & 11 & $3,0 \%$ & $2,5 \%$ \\
\hline Total & 367 & $100,0 \%$ & $82,7 \%$ \\
\hline
\end{tabular}

* Internationalisation notamment à partir de Maltreurop et de Nutrixo.

** Internationalisation à partir de Laïta, filiale avec Terrena et la coop. de Ploudaniel. 
Les implantations se situent:

- en Europe: Espagne, Allemagne, Portugal, Belgique, Ukraine;

- en Asie: Chine;

- en Amérique latine: Argentine.

Les implantations de Nutrixo sont pratiquement dans les mêmes pays.

\section{Tereos (sucre), 1,600 milliard d'euros (2002-2003)}

Ce groupe sucrier, premier groupe français et deuxième mondial, a repris avec quelques autres coopératives sucrières l'essentiel des usines du premier groupe sucrier français, Béghin-Say.

Ce groupe est aujourd'hui présent en Suisse, en République tchèque, en Hongrie, en Espagne et surtout au Brésil.

La stratégie d'internationalisation du groupe consiste à récupérer les marchés internationaux, notamment par les filiales brésiliennes, pour faire face aux changements de la Pac dans les prochaines années.

\section{Les semenciers}

- In Vivo: 2,727 milliards d'euros.

- Limagrain: 0,933 milliard d'euros.

- Euralis: 0,868 milliard d'euros.

- Maïsadour : 0,566 milliard d'euros.

Ces groupes du top 11 ont une internationalisation essentiellement tournée vers les semences, même lorsqu'ils sont plus diversifiés (comme Maïsadour, Euralis ou In Vivo).

Ces quatre groupes cumulent cent opérations.

Le plus internationalisé des quatre est Limagrain, qui, sans être au top des leaders mondiaux des semences, figure en bonne place.

La Cecab (marque D’Aucy), pour conserver sa place européenne, notamment face à Bonduelle, intensifie son internationalisation.

\section{Eléments de synthèse sur l'internationalisation des coopératives}

L'internationalisation, la délocalisation ou l'importation de produits étrangers concurrents constituent rarement des décisions de premier choix pour une coopérative. En effet, ces différentes stratégies apparaissent souvent comme non pertinentes par rapport aux finalités de l'entreprise coopérative centrées sur la valorisation du produit. Cette finalité se manifeste par plusieurs indicateurs:

- le prix du produit;

- le revenu de l'adhérent ou l'optimisation des "droits à produire".

Les stratégies, très nombreuses, de filiales commerciales à l'étranger sont cohérentes et facilement explicables à l'adhérent. Elles ne sont que le prolongement de l'activité classique d'exportation.

Dans les exemples cités, certaines stratégies sont cohérentes avec le but de la coopérative, à savoir faire une partie du processus de transformation à l'étranger pour conserver la filière compétitive (exemple: cas noix); elles peuvent même être la condition de survie de la filière et donc de la 
finalité de la coopérative. Cette fonction délocalisée peut être effectuée par une filiale de la coopérative.

La stratégie de franchise est également facile à expliquer s'il s'agit de produits peu exportables dans de bonnes conditions. Les avantages financiers pour l'adhérent apparaissent souvent faibles, mais comme l'investissement de départ est peu élevé, la décision n'est pas trop difficile à prendre (exemple: Yoplait).

Dans le cas de Malteurop, on peut retrouver une cohérence par rapport aux finalités de la coopérative, mais la démonstration est plus complexe et pas toujours convaincante pour l'adhérent. Si la filiale est bénéficiaire, la perspective de remontée des dividendes peut faciliter la prise de décision par l'agriculteur, mais le bilan global de l'opération sur le plan économique est toujours difficile à faire entre la baisse éventuelle de valeur pour l'usager et l'accroissement éventuel de valeur pour l'actionnaire. Il s'agit dans ce cas de suivre le client brasseur.

Si la filiale étrangère est déficitaire, l'argumentation est encore plus difficile. En résumé, dans ce cas de figure, la stratégie d'internationalisation est souvent perçue comme un moindre mal et argumentée par les dirigeants de cette façon pour sauver l'essentiel, c'est-à-dire le maintien ou la moindre baisse de la production des adhérents.

Ce type d'investissement à l'étranger se fera le plus souvent sur fonds propres du groupe coopératif ou sur fonds empruntés, plus rarement avec une nouvelle souscription des adhérents ciblée sur cette stratégie.

Notre étude conduite par l'Essec-Imia sur huit années (1996-2003) dans l'agroalimentaire en France montre l'augmentation importante des manœuvres d'internationalisation dans les entreprises ayant le capital comme facteur risqué (entreprises capitalistes); ceci est cohérent avec la globalisation des marchés. Par contre, les entreprises ayant comme facteur risqué le produit (les coopératives) représentent une faible part dans les opérations d'internationalisation. Cette observation est la conséquence, toutes choses égales par ailleurs, de la différence sur les facteurs risqués. Quelques autres variables peuvent intervenir, mais de façon secondaire (moyens financiers, notamment).

\section{Quelles finalités demain?}

\section{De la valeur pour l'usager à la valeur pour l'actionnaire}

En effet, dans un groupe coopératif complexe internationalisé, le mode de rémunération de l'adhérent évolue, notamment dans le cas où il y a des partenaires capitalistes (banques ou industriels). La valeur de la coopérative pour l'usager n'est plus le seul critère; apparait alors la valeur du groupe coopératif pour l'actionnaire (voir la définition des concepts).

On pourrait concevoir, et c'est déjà le cas aujourd'hui pour quelques groupes coopératifs, que la valeur pour l'usager stagne ou régresse, mais que le relais soit pris par la valeur pour l'actionnaire, notamment à partir des stratégies d'internationalisation (par exemple, Limagrain, Tereos...).

Cette valeur pour l'actionnaire peut se manifester de plusieurs manières: dividendes à partir du capital détenu dans la société coopérative mère, mais 
également à partir du capital que l'adhérent peut mettre directement dans les filiales, ce qui lui permet en droit français d'avoir également accès dans ce deuxième cas aux plus-values.

Ainsi, au lieu de définir la finalité à partir de la recette du produit, l'adhérent peut regarder la recette globale: revenu du produit (valeur pour l'usager) plus revenu du capital (valeur pour l'actionnaire).

La bonne stratégie d'internationalisation sera celle qui maximisera cette double valeur à moyen terme. Il s'agit là d'un calcul complexe, car dans le cas de l'internationalisation, ces deux valeurs peuvent être en partie opposées (par exemple, accroissement de la valeur pour l'actionnaire en poussant la délocalisation au détriment de la production locale [valeur pour l'usager]).

Dans la pratique stratégique des groupes coopératifs, devant la difficulté d'effectuer une consolidation économique de l'avantage de l'internationalisation et de ses éventuels effets pervers pour l'usager, le groupe coopératif limitera souvent son analyse à la rentabilité du projet et aux retombées éventuelles sous forme de dividendes pour la mère et (ou) pour les adhérents. Les effets sur la valeur pour l'usager sont évalués en tendance, mais difficilement chiffrables, et en faisant un raisonnement en termes de moindre mal. Dans le cadre de marchés de plus en plus globalisés et de concurrents internationalisés, les choix coopératifs en matière d'internationalisation seront souvent difficiles à prendre; il s'agit cependant d'un enjeu important pour les coopératives au début du XXI siècle.

Remarque: les principes coopératifs sont bien sûr «bousculés " par ces stratégies et ces modes de répartition du surplus.

\section{Les coopératives, un atout pour le développement durable face à la mondialisation}

Dans cette approche, nous nous limiterons à un aspect important du développement durable, à savoir l'ancrage territorial. Les autres aspects ne seront pas traités.

Dans ce contexte de mondialisation, on aurait pu croire à une perte de parts de marché des coopératives. Or, depuis 1997, elles en gagnent.

Aujourd'hui, à peu près 2 à $3 \%$ de parts de marché ont été gagnées sur l'industrie capitaliste agroalimentaire en France. En effet, avec la mondialisation, les opportunités de profits ont évolué sectoriellement et géographiquement. Les entreprises capitalistes s'adaptent à ces évolutions en modifiant leurs portefeuilles d'activités. Les coopératives reprennent les actifs délaissés pour conserver les activités sur le territoire. Quelques exemples...

- Béghin-Say: la rentabilité devrait diminuer à partir de 2006, avec la réforme du règlement sucrier. Les fonds de pension majoritaire retirent leurs capitaux du secteur et les coopératives sucrières ont racheté ce groupe. - GMP (Grands Moulins de Paris), qui était entre les mains notamment de la société d'assurances Axa; même stratégie de dégagement: les coopératives de céréales reprennent avec les salariés de GMP. 
- Le foie gras: là encore, le secteur bancaire n’a pas eu les résultats escomptés et a effectué un dégagement. Aujourd'hui, trois groupes coopératifs ont repris l'essentiel des actifs.

Ces quelques exemples illustrent la stratégie de compensation des coopératives pour maintenir sur le territoire français des productions et des emplois qui, sans ces reprises, pourraient être délocalisés.

On voit ainsi le rôle déterminant des coopératives dans les territoires. Par ce biais, les coopératives participent au développement durable.

Il existe aussi des spécificités juridiques qui permettent d'éviter des OPA. Cela n'a cependant pas totalement empêché des groupes capitalistes de reprendre des actifs coopératifs suite à des difficultés (ULN, Arcadie...) et plus récemment le Cabanon, repris par un groupe chinois dans les conserves de tomates.

L'ancrage territorial constitue quand même une spécificité coopérative qui correspond bien à une logique de long terme.

Nous pensons que les coopératives sont plus qu'hier nécessaires pour l'agriculture. Hier, de 1960 à 2000, elles étaient moins indispensables en raison d'une Pac protectionniste. Aujourd'hui et demain, elles deviennent déterminantes face aux risques de délocalisations, conséquence de la mondialisation.

\section{Conclusion}

(9) Notamment maintien de certains prélèvements ou d'aides compensatoires (voir soutiens en tableau 1).
En conclusion, dans cet univers perturbé, il faut poursuivre la réflexion sur la trajectoire et le sens à donner aux entreprises coopératives.

Au terme de cette approche, on peut affirmer qu'une large majorité des coopératives est indispensable pour conserver les activités et les emplois de façon durable. Face aux déréglementations en cours, les coopératives sont des structures pertinentes et adaptées pour le XXI siècle, mais il convient d'être vigilant pour que la gouvernance des coopératives, et notamment des groupes complexes, maintienne le cap sur le long terme.

L'internationalisation des coopératives est parfois nécessaire, mais il convient de la resituer par rapport aux finalités de la coopérative, laquelle peut aussi évoluer.

L'ancrage sur le territoire restera cependant déterminant pour l'agriculteur, même si ce dernier peut aussi se délocaliser.

Néanmoins, la coopérative, de par ses finalités (valorisation du produit d'abord) et son organisation (principe de dévolution d'actif), constitue une forme d'entreprise allant dans le sens du développement durable.

Ce n'est cependant pas une assurance tous risques et l'organisation coopérative ne pourra pas empêcher toutes les délocalisations de production. Les exemples évoqués de délocalisation, dans les légumes et les poulets, sont là pour confirmer ce risque. Une bonne organisation du commerce mondial et une politique européenne adaptée ${ }^{(9)}$ sont nécessaires compte tenu des différences de coût des produits au niveau mondial. 


\section{Bibliographie sélective}

Agra-Alimentation (revue), 1996 à 2004.

Agra-Industrie (revue).

Berthelot Jacques, L'agriculture talon d'Achille de la mondialisation, L'Harmattan, 2001.

Caby J. et Hirigoyen G., La création de valeur de l'entreprise, Economica, 1997.

Cepiil, L'économie mondiale, La Découverte, 2004.

Côté Daniel, Les holdings coopératifs, De Boeck Université, 2001.

Cyclope, Les marchés mondiaux, Economica, 2003.

Déméter, Economie et stratégie agricole, Armand Colin, 2004.

Deshayes Gérard, Logique de la coopération et gestion des coopératives, Skippers, 1988.
Hubert Jacques et Colson Jean-François, Rapport du Conseil supérieur d'orientation de la coopération agricole française, février 2004.

Touzard Jean-Marc et Draperi Jean-François (coord.), Les coopératives entre territoires et mondialisation, "Les Cahiers de l'économie sociale ", $\mathrm{n}^{\circ}$ 2, IES-L'Harmattan, 2003.

Insee, Tableau de l'économie française, 20032004.

Krugman Paul R., La mondialisation n'est pas coupable, La Découverte, 2000.

Koulytchizky Serge et Mauget René, " Le développement des groupes coopératifs agricoles depuis un demi-siècle: à la recherche d'un nouveau paradigme ", Recma, n² 287, 2003. Mauget René et Forestier Michel, «De la coopérative au groupe coopératif agroalimentaire ", Recma, nos 278 et 279, 2000, 2001. 